\title{
The agency of artefacts: Socio- ideological functionality and the long-necked spearthrowers of Mirarr Country, northern Australia
}

\author{
John A. Hayward
}

\section{Introduction}

The seemingly symbiotic relationship between spears and spearthrowers in the history of Australian Aboriginal technologies is not as equal as it may appear. Spears can, and have, happily survived without spearthrowers, whereas, as instruments of propulsion, spearthrowers appear to be solely dependent upon spears for their raison d'être. The first people to arrive in Australia probably came with spears (see also Davidson 1934:42), but it was not until many thousands of years later that spearthrower technology was introduced into the region (see also Davidson 1936). Some parts of Australia such as Tasmania, Melville Island, parts of the Queensland coast and possibly southern Queensland, northern New South Wales and eastern South Australia never adopted the technology (Cundy 1989:1; Davidson 1936:453). In the rock art of Arnhem Land, some of the earliest depictions of human figures with material culture, known as 'Dynamic Figures', only launched their spears by hand, with the first images of spearthrower-like implements appearing towards the end of the Dynamic Figure phase as a hooked stick that could have functioned as either a fighting pick or a prototype spearthrower (Lewis 1996:17), and may have functioned as both in those transitional times. Rock art images suggest that these implements were first used sometime after the Pleistocene/Holocene transition, more than 40,000 years after colonisation of the continent, but the images do not tell us if the new technology was a local invention, or introduced from elsewhere through diffusion as proposed by Davidson (1936:446).

It is generally considered or assumed that since its adoption at the end of the period of Dynamic Figures art or slightly later in Arnhem Land, the spearthrower was instrumental in increasing the power and efficiency of spear throwing (see Allen and Akerman 2015:82-83; Cundy 1989:16). However, some rock art images from the northeast Kakadu region testify to another, independent role for the implement. 

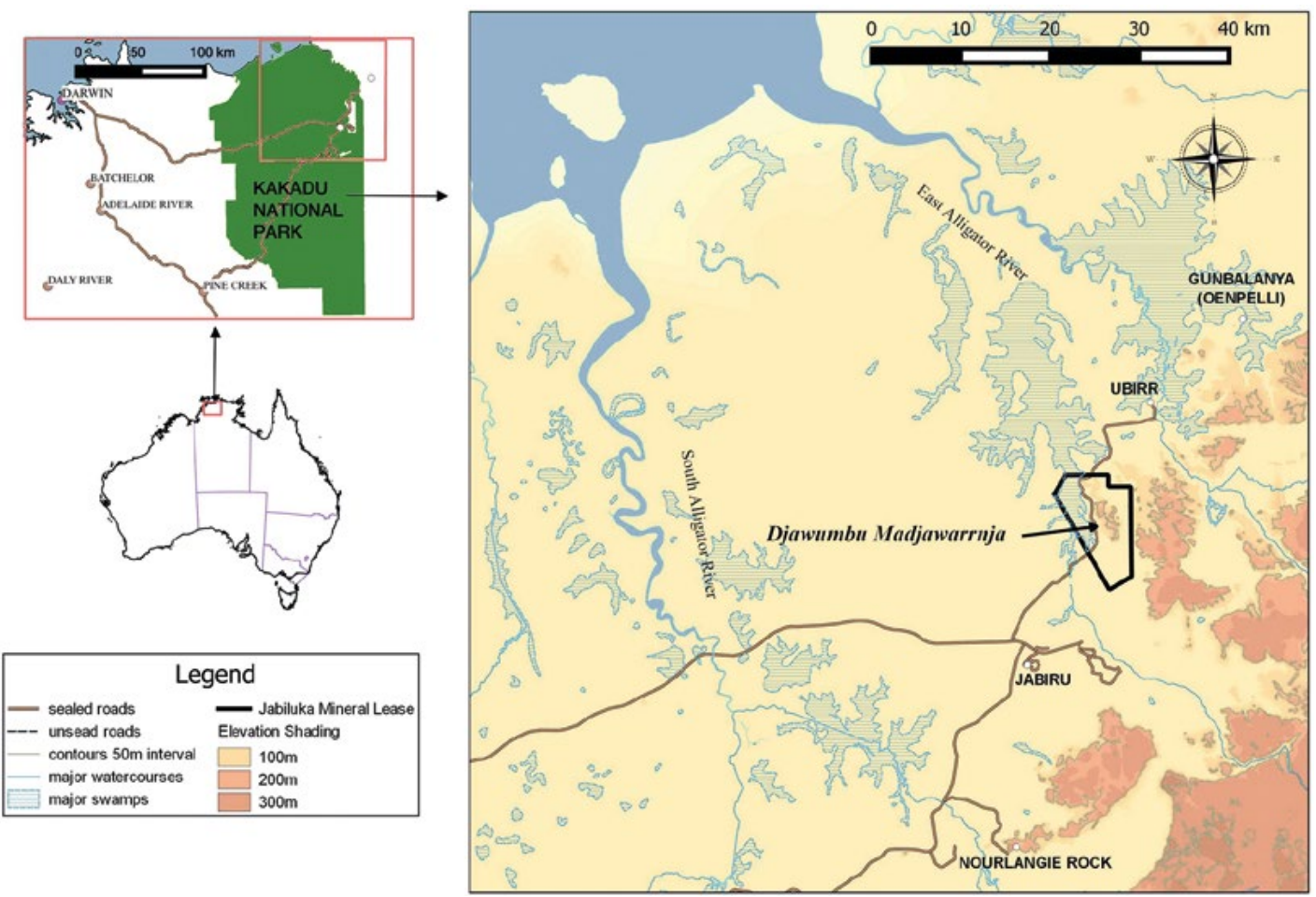

Figure 4.1 Location of the Djawumbu Madjawarrnja massif in relation to the township of Jabiru and the Jabiluka mineral lease.

Source: Phil Davill.

\section{Long-necked spearthrowers in the rock art of Arnhem Land}

This chapter focuses on images of long-necked spearthrowers, a particular type of spearthrower depicted in the rock art of a discrete region of western Arnhem Land. These images were documented during the 2012-2014 field seasons of the Mirarr Gunwarddebim (rock art) project in the traditional Country of the Mirarr people in northeastern Kakadu National Park. The study focused on the Djawumbu Madjawarnja massif located within the Jabiluka mineral lease a few kilometres north of the Jabiru township (Figure 4.1). The massif has a high concentration of rock art; during the first three years of the Mirarr Gunwarddebim project, 528 cultural sites were documented within an area approximately $5 \times 2.7 \mathrm{~km}$ in size.

One of the prevailing views relating to Aboriginal artefacts includes the idea that the design variation of implements be analysed through their function in the technological sub-system, where form follows function (Cundy 1989:3). Long-necked spearthrowers, however, represent an unusual and distinctive graphic within the rock art landscape of this region, because they appear as functional spearthrowers used to launch a range of spear types, but the elaboration, form and variation of their design elements give the impression of being functionally impractical. It is due to this apparent impracticality that these implements have been analysed within the social, ideological and symbolic framework of rock art, rather than as technological items.

It is the extended central shaft of the implement protruding like a long neck from its body that unites this spearthrower type under the common banner of 'long-necked spearthrowers' (Figure 4.2). This implement type has also been described by Lewis (1988) and mentioned by Chaloupka (1993:150) as 'long-necked spearthrowers', and Chaloupka (1993:217) as 'complex spearthrowers'. In all cases, the central shaft of the spearthrower appears to be a stout stick with 
a hooked end for articulation with a spear. There is generally a handle, or enlarged form at the proximal end, which can take on a number of different shapes; some appear to have tassels on either or both the proximal and terminal ends. The central body of the implement takes on a variety of shapes that are often depicted as disproportionally large forms, sometimes with distinctive patterning inside them.

In his 1988 monograph, Darrell Lewis proposed a chronology for Arnhem Land rock art based principally on his observations of technological change of implements such as boomerangs, spears and spearthrowers in paintings, but also backed up by other chronological markers, such as cultural and stylistic conventions, broad environmental changes and faunal extinctions. Lewis's methodologies were very much influenced by Eric Brandl, an earlier researcher who is credited not only with recognising Mimi art as having early and late periods (Brandl 1973), but also as observing the technological transition within those periods from hand-thrown spears to spearthrower-launched spears (Lewis 1988:16). Some of the earliest human forms depicted in the art (Dynamic Figures) are devoid of spearthrowers but are commonly associated with boomerangs, a type of implement that subsequently became quite scarce in that same region's rock art. Lewis's first chronological period, the boomerang period, was thought to have lasted until at least 9000 years ago, at which point it morphed into the hooked stick period when both boomerangs and hooked fighting sticks were concurrently depicted. By Lewis's estimate, approximately 6000 years ago the first unambiguous spearthrower types are seen. These broad spearthrowers', which along with basic 'cylindrical spearthrowers' and a wide variety of new spear types begin to appear in the rock art that defines the broad spearthrower period, are thought to have begun no more than 6000 years ago, lasting to at least 1000-2000 years ago. The subsequent long spearthrower period, which Lewis estimates to have spanned from a maximum of 2000 years ago to ethnographic times, was named after the introduction of a type of spearthrower that is longer and thinner than broad spearthrowers and that is defined by a notch functioning as a handle at its proximal end (see Figure 4.2).

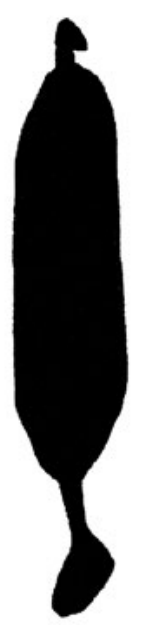

Broad
spearthrower

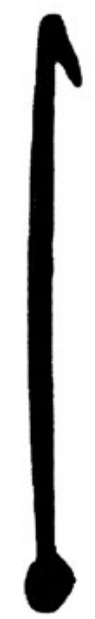

Cylindrical spearthrower

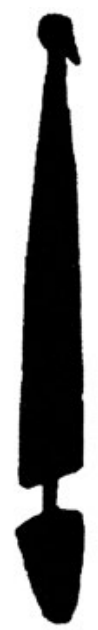

Long-notched lath spearthrower

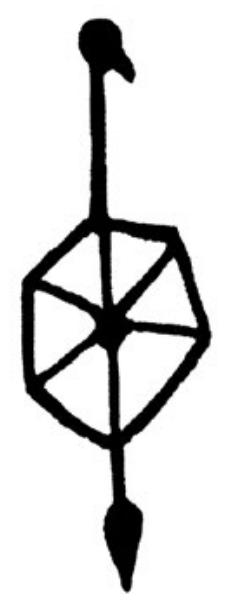

Long-necked spearthrower

Figure 4.2 Four typical spearthrower types recorded during the Mirarr Gunwarddebim project (not to scale).

Source: John Hayward. 
According to Lewis (1988:111), long-necked spearthrowers appear in rock paintings of western Arnhem Land during the transition between broad to long spearthrowers. Lewis's (1988:62) assessment is as follows:

The long-necked spearthrower does not appear to have an ethnographic parallel anywhere in Australia. The possibility of artistic license must always be considered, but in this instance the very fine line-control and carefully executed patterns inside the round or squared section of some examples suggest that its shape is being accurately rendered and that these are depictions of an archaic spearthrower type. Several examples bear very similar designs inside their borders which suggests that they are not mere infill patterns. Whether these patterns represent part of the construction of the spearthrower or a painted or incised design is a problem for future research.

The variety of shapes apparent among long-necked spearthrowers suggests that they were produced during a period of experimentation with spearthrower/spear technology. If this is so it is possible that long-necked spearthrowers were only produced for a short period and were not adopted throughout the Arnhem Land region.

Here, I reassess the chronology of Lewis's broad and long spearthrower periods in relation to the abundance of long-necked spearthowers observed in motifs recorded during the Mirarr Gunwarddebim project. Along with these new field observations, two artefacts recently recorded from an ethnographic collection (see below) have possible implications for the age of this type of spearthrower, and therefore for the age of artworks that contain such artefacts.

A total of 107 individual paintings of, and scenes with, long-necked spearthrowers were recorded during the first three field survey seasons of the Mirarr Gunwarddebim project. Some scenes depict multiple spearthrowers, giving a total of 138 long-necked spearthrowers counted from photographs taken at 67 rock art sites. Of the 667 paintings of spearthrowers of various types recorded from all sites in the Djawumbu region, long-necked spearthrowers represent 20.7 per cent, or over one-fifth of the total (long-notched lath spearthrowers $=38.2$ per cent; cylindrical spearthrowers $=22.6$ per cent; broad spearthrowers $=7.5$ per cent) (Figure 4.2). My use of the term 'long-notched lath spearthrower' throughout this chapter is equivalent to Davidson's (1936) 'North Australian type', Cundy's (1989) notched 'lath spearthrower' and Lewis's (1988) 'long (notched lath) spearthrower', although Lewis oscillates between 'long notched' and 'long (notched lath)' spearthrower.

The main attribute of long-necked spearthrowers is that, unlike the broad, cylindrical and longnotched lath spearthrowers, each of which displays considerable graphic conformity, individual representations of long-necked spearthrowers are generally quite different from each other. Of the 138 recorded cases, 78 representations ( 57 per cent) have unique characteristics, with the remaining exhibiting varying degrees of difference to each other (Table 4.1). This uniqueness or individuality of each long-necked spearthrower in the rock art can be expressed in a number of ways, including the shape of the body, the infill pattern of the body, the shape of the handle, the shape of the hook and/or the addition of tassels. The 78 renditions of long-necked spearthrowers recorded during this survey are shown in Figures 4.3-4.5. Another 22 examples recorded by previous researchers from parts of western Arnhem Land outside the Jabiluka area (Figure 4.6) suggest that the general distribution of this spearthrower type is restricted to the northwest section of what is today Kakadu National Park, and seems to follow similar distribution patterns to other motifs such as Mountford Figures and Energetic Figures (see Haskovec 1992; Lewis 1996:13; Taçon 1989:136). Chaloupka (1993) recorded a number of sites with long-necked spearthrowers at Injalak near Oenpelli, as has Lewis (1988) at Cannon Hill that appears to represent the northernmost reach of this motif. Both Welch (1997) and Lewis (1988) have recorded long-necked spearthrower motifs at Nourlangie, as has Brandl (1973:48) at Mt Gilruth, the latter being the southernmost mention of this type of spearthrower. 


\section{Sub-types of long-necked spearthrowers}

For purposes of classification and discussion, the diversity of shapes among long-necked spearthrowers can be divided into four main sub-types: Type A: rectilinear; Type B: curvilinear; Type C: concave-sided; Type D: multi-sided (Table 4.1). These four sub-types represent morphological variations on the common theme of spearthrowers with long necks, although whether or not this four-part division was recognised by the artists is unknown. As with many classification systems, some paintings are borderline cases that could have fitted into more than one category but have been allocated the sub-type considered 'best fit'.

Table 4.1 Numbers of long-necked spearthrower paintings, by sub-type.

\begin{tabular}{|l|c|c|c|}
\hline Long-necked spearthrower types & Number of paintings & \multicolumn{2}{|c|}{ Unique examples } \\
\hline A: Rectilinear & 24 & 20 & $83 \%$ \\
\hline B: Curvilinear & 86 & 39 & $45 \%$ \\
\hline C: Concave-sided & 16 & 12 & $75 \%$ \\
\hline D: Multi-sided & 12 & 12 & $100 \%$ \\
\hline Total & 138 & $\mathbf{7 8}$ & $\mathbf{5 7} \%$ \\
\hline
\end{tabular}

Source: Author's data.

There are more paintings of curvilinear long-necked spearthrowers than of all other sub-types put together, and the curvilinear sub-type is more standardised than any other (Table 4.1). Some spearthrowers from a given sub-type appear in scenes where large groups of human figures are engaged in possible ceremonial, conflict or other activities. No two spearthrower depictions are exactly the same within any sub-type; by definition, those that are considered 'similar' to each other have fewer degrees of difference than those classed as 'unique'. The examples depicted in Figures 4.34.5 show clear morphological differences from each other and are those classed as 'unique'. Of these 78 unique designs, 18 are identified as the models for other 'similar' cases found in either the same, or in different, paintings.

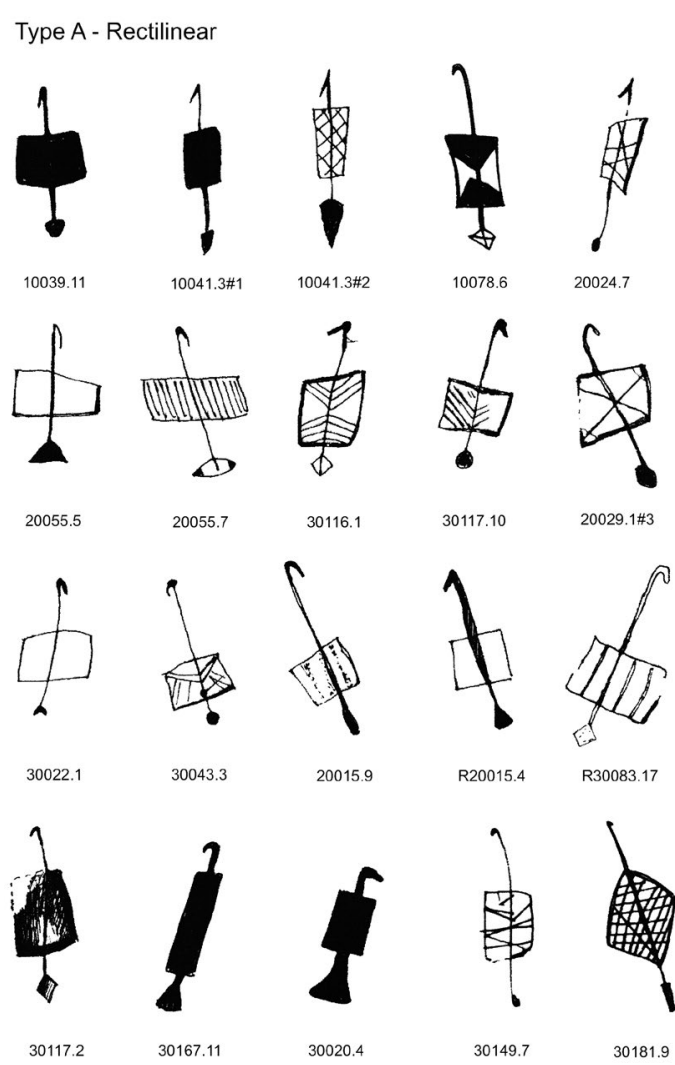

Figure 4.3 Type A (rectilinear) long-necked spearthrowers from Mirarr Country. Individual codes refer to site and image numbers. Source: John Hayward. 


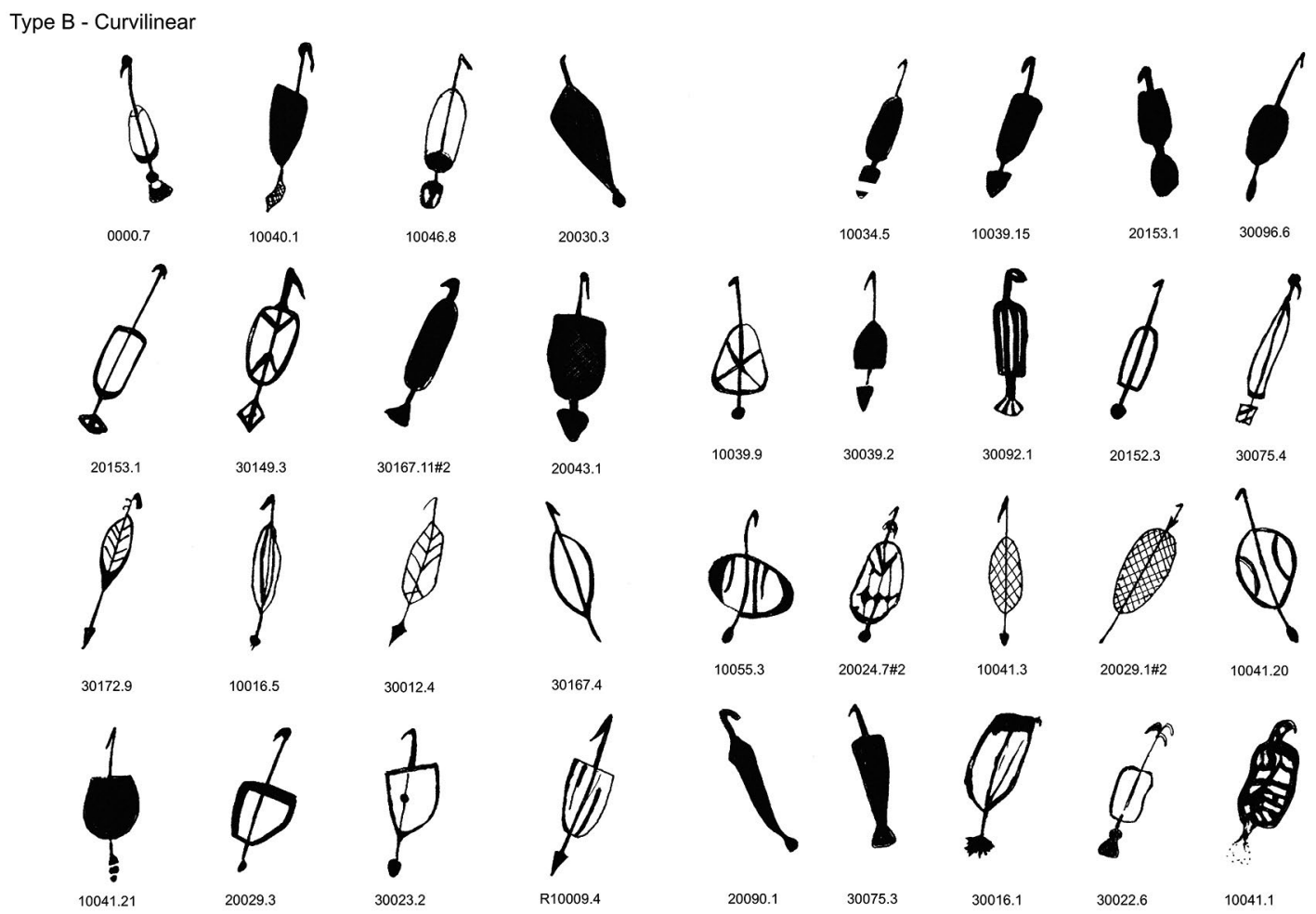

Figure 4.4 Type B (curvilinear) long-necked spearthrowers from Mirarr Country.

Individual codes refer to site and image numbers.

Source: John Hayward.

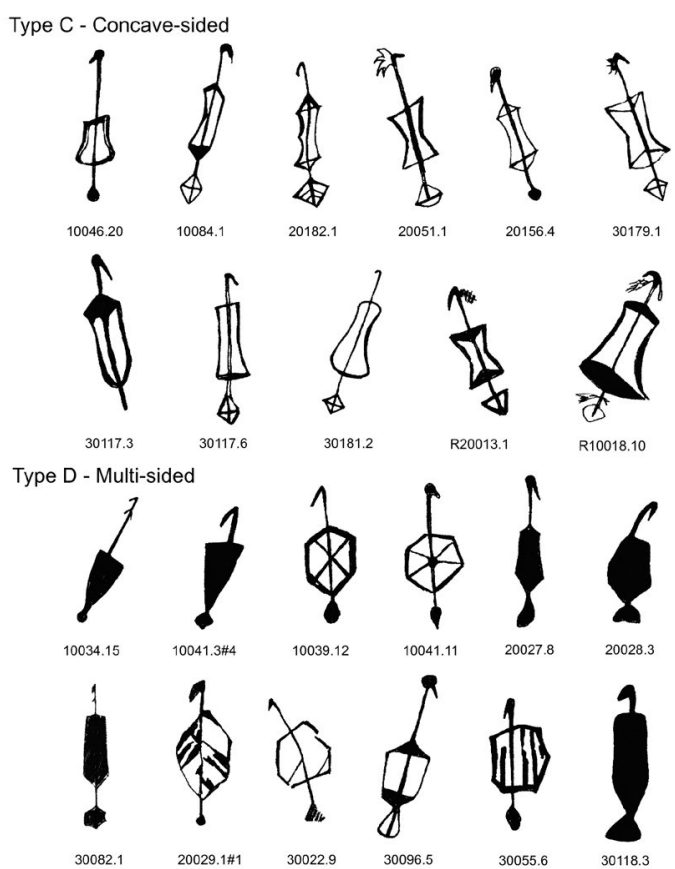

Figure 4.5 Type C (concave-sided) and Type D (multi-sided) long-necked spearthrowers from Mirarr Country.

Individual codes refer to site and image numbers.

Source: John Hayward.

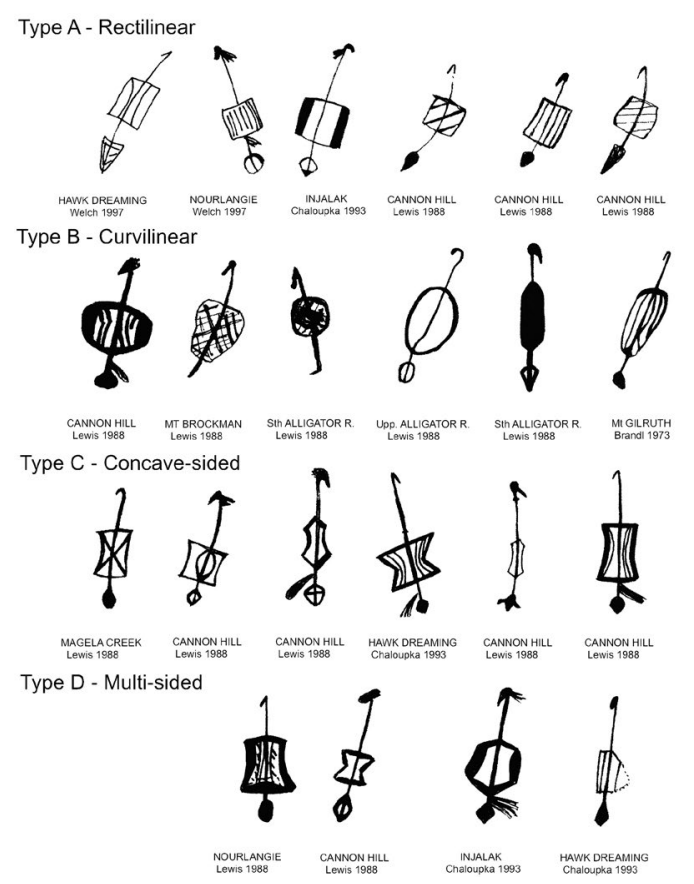

Figure 4.6 Long-necked spearthrowers from other locations as recorded by Brandl (1973), Lewis (1998), Chaloupka (1993) and Welch (1997). Source: Drawing by John Hayward after Brandl (1973), Lewis (1998), Chaloupka (1993) and Welch (1997). 


\section{Case studies}

The following examples illustrate different aspects of long-necked spearthrower depictions in western Arnhem Land rock art. Some of the images have been digitally enhanced using the DStretch program and inked-in to highlight the artworks.

\section{Site 20029}

The three male human-like figures in Figure 4.7 have been superimposed by some yellow elongated human-like figures that have since faded. Two of the male figures have straight, widely spread legs and are superimposed over a third male figure with slightly curved near-horizontal legs. All three are superimposed over a number of other weathered black human-like figures. The leading male figure uses a multi-sided spearthrower with an internal design to launch a uni-serial square-barbed spear. This figure also carries a second, different type of uni-serial spear, traditionally called bokko (Chaloupka 1993:146), in the other hand along with a small goose-wing fan and wears a small tasselled headdress. Goose-wing fans were made from the wings of the magpie goose and are typical of Chaloupka's $(1984,1993: 185)$ recent 'Freshwater Period' of the past 1500 years, when large wetland areas developed along the floodplains near the base of the Arnhem Land escarpment, attracting higher numbers of fish species and water-birds than previously.

One of the leading male figure's two companions has a three-pronged barbed spear being launched by a curvilinear long-necked spearthrower with a distinctive cross-hatched internal pattern. The more weathered curved-leg male figure is launching a bokko with a rectilinear spearthrower that has an indistinct internal design. This latter male figure has a pronounced headdress and wears a small bag around his neck, perhaps a 'biting bag' (for an ethnographic example, see Hamby 2010:261-264).

The two male figures with widely spread angular legs have 'hairy' upper arms. Chaloupka (1993:217) notes of such figures: 'Nalbidji figures, the "hairy armed" people, are clearly human beings and are depicted performing traditional activities. They occur in individual representations and in compositions, and seem to represent a local group of people'. Such figures conform to a number of other humanlike painting types such as Mountford Figures (Haskovec 1992; Lewis 1996:13) and Energetic Stick-Figures (Taçon 1989:136), each of which has a limited spatial distribution in much the same area along the northwestern margin of the Arnhem Land plateau. Chaloupka thought that the hairy arms on such figures represent feathers or pelts tied to the arms as a form of decoration. Images of Nalbidji often depict long-necked spearthrowers along with capelike adornments and tasselled spears as part of their repertoire of material culture items (Chaloupka 1993:217).

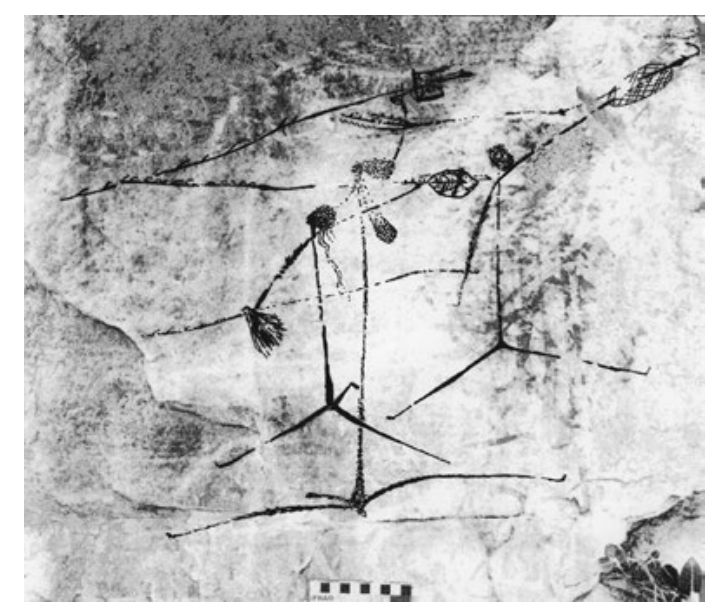

Figure 4.7 Three male figures with morphologically singular long-necked spearthrowers, from site 20029.

Source: Photograph by John Hayward. 
The male figures in the painting scene discussed above appear to represent a seemingly active and unified force, all moving in one direction with spears ready, albeit each carrying a different kind, or set, of spears and spearthrower. In this sense, each male figure identifies as an individual personality through his associated items of material culture, rather than being reduced to an undifferentiated member of a group.

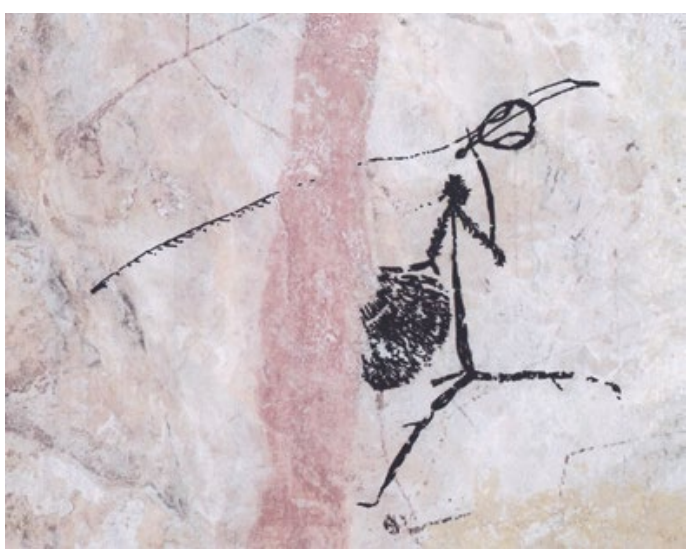

Figure 4.8 Individual human figure with a longnecked spearthrower and goose-wing fan, from site 10041.

Source: Photograph by Inés Domingo Sanz, enhancement by John Hayward.

\section{Site 10041}

The human-like figure depicted in Figure 4.8 is holding a curvilinear long-necked spearthrower with a very distinctive internal design. The depiction of the central section of this spearthrower allows the shaft of the spear to be seen through the body of the spearthrower. This Nalbidji hairy-armed figure has a more solid body and limbs than the stick-figures of Figure 4.7 and carries a large goose-wing fan.

\section{Site 10039}

All three figures in Figure 4.9 are males with accentuated penises, and have widely spaced but not horizontal legs that suggest a walking action. Two hold rectilinear long-necked spearthrowers and are superimposed over a number of weathered black stick-figures, one of which also holds a long-necked spearthrower. The largest and most dominant human-like male figure is heavily adorned with arm pelts, tassels and a body covering that simultaneously resembles a long cape, a goose-wing fan and a basket.

The largest male figure in Figure 4.9 wields a large, square-shaped rectilinear long-necked spearthrower that either has a pronounced hafting or a short tassel and is used to launch a bokko spear. It is unclear if the square-shaped bodies of the two rectilinear long-necked spearthrowers on this panel are internally decorated. What is clear, however, is the stark contrast between the elaborate adornment of the largest, dominant male figure and that of the other male figure with a squareshaped rectilinear long-necked spearthrower, which has none. Below these two figures is the third male figure who carries a hooked stick-shaped cylindrical spearthrower, a long string bag around his neck and an unusual, pronounced headdress or tasselled hair. As with many other paintings containing long-necked spearthrowers, the emphasis is on the depiction of individual differences rather than on undifferentiated conformity.

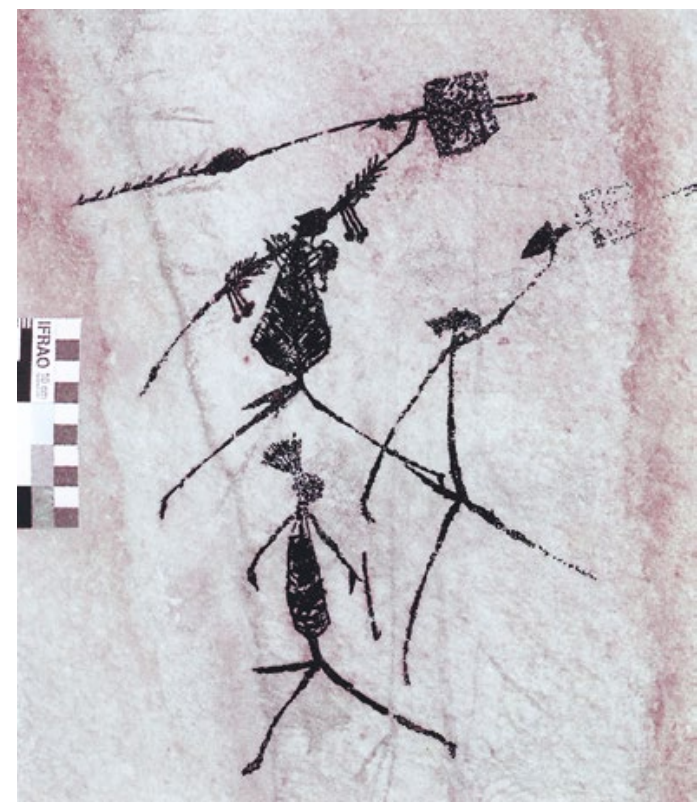

Figure 4.9 Group of three male figures, from site 10039 .

Source: Photograph by Inés Domingo Sanz, enhancement by John Hayward. 


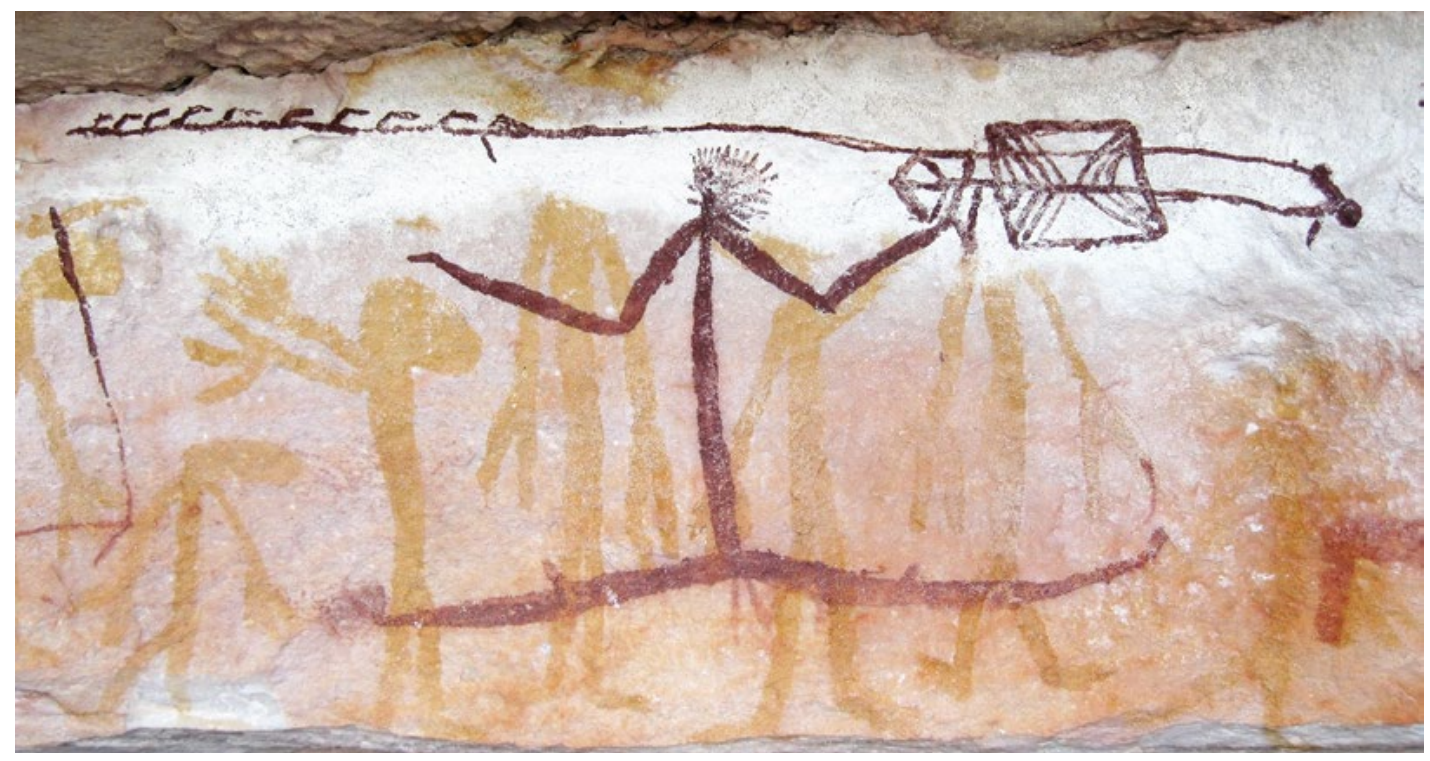

Figure 4.10 Human-like figure with near-horizontal legs launching a spear with a rectilinear longnecked spearthrower, from site 30116.

Source: Photograph by John Hayward.

\section{Site 30116}

The human-like figure in Figure 4.10 is distinctive for its spiky hair and energetic stance. The image is painted in a deep red pigment over yellow pigment human figures at a site with commanding views over Magela Creek to the southwest of the Madjawarrnja massif. This human-like figure is launching a square-barbed spear with a rectilinear long-necked spearthrower that has a unique internal design and a diamond-shaped handle. The square body of the implement sits closer to the handle than the exaggerated large 'hook', and its depiction appears as an open construction or see-through representation that allows us to see the spear shaft behind it, although alternatively the spear could be held in front of it. The human-like figure in this painting exudes an air of confidence and command over 'his' body and implements; one finger controls the balance of the spear while the other handles the spearthrower with a sense of deftness and assurance. The contrast between this finely painted image and the somewhat less-refined yellow human-like paintings that underlie it is quite dramatic, almost as if the artist chose this position to highlight the differences. The fresh appearance of the red pigment used in this painting over a salt crust on the rock suggests is a very recent painting, further adding to the mystery of what happened to the long-necked spearthrower in the 'recent' history of this region.

\section{Site 10040}

The group of eight stick-figures in Figure 4.11 is, in my opinion, one of the highlights of rockpainting visual composition in the Jabiluka region. Eight male figures, painted in light red, move as one in a choreographed performance of unity, individuality and ambiguity. The figures and the implements have equal importance in the composition, emphasising the relationship between them and the role of each. Four of the figures carry curvilinear long-necked spearthrowers and four others carry another kind of spearthrower previously unrecorded in this region. These latter spearthrowers appear to be very long implements with the characteristic hook at the distal end and an enlarged proximal end that could act as a counterweight balance to the length. These implements are held by their carriers at the intersection of the weighted end and the shaft. No spears are depicted in this image, allowing the spearthrowers an autonomous significance. 
The leading and trailing figures are differently adorned to all six central figures. The leading figure wears arm pelts while the trailing figure wears a cape of sorts. The leading figure also carries three extra stick implements that could be fighting sticks with enlarged ends. Three of the trailing figures raise their long-necked spearthrowers in the air; the front three raise their spearthrowers higher still. The middle couple, in defiance of the group, behave a little differently, the front of the two holding both arms down with a curvilinear spearthrower in one hand and another stick implement in the other, and the second of the two raising a long spearthrower, but lower than the rest.

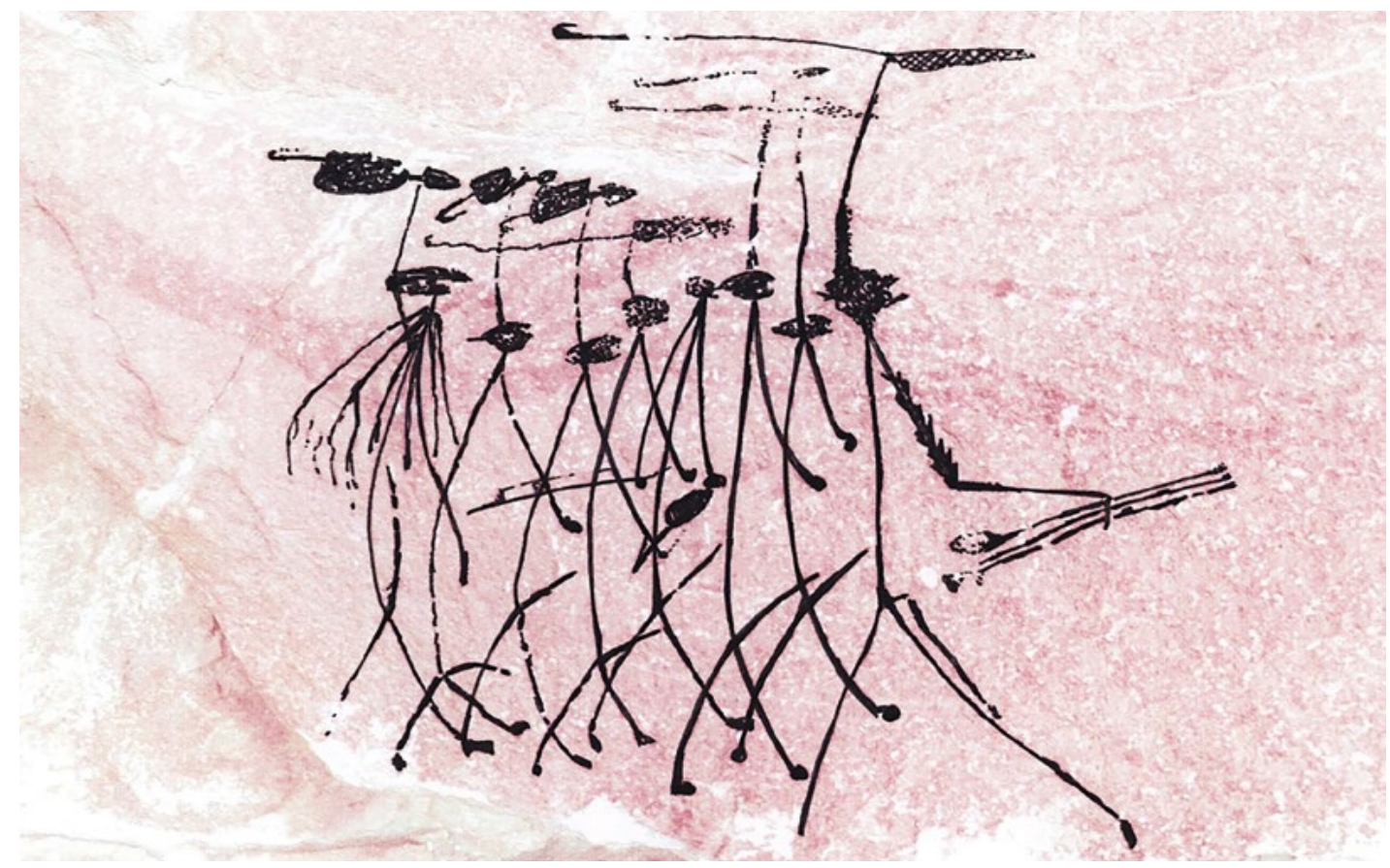

Figure 4.11 Group of eight stick-figures holding two types of spearthrowers, engaged in choreographed movement, from site 10040.

Source: Photograph by Inés Domingo Sanz, enhancement by John Hayward.

Despite these anomalies to conformity, there is a rhythm to the composition that is pronounced by the bend of the torsos, the alignment of the heads and the angles of the penises which follow through on the line of the legs, except for the front figure whose alignment of the 'broken' penis is replaced by the alignment of the fighting sticks. The counterpoints to this unified rhythm are the three levels of raised and lowered spearthrowers above the heads and among the bodies. Overall, the whole image expresses a complex unity composed of differentiated individuals and artefacts, with an added masterly touch of ambiguity, the essence of all good art making, according to Zeki (2006:243). This scene also embodies the concept of 'methexis' where, through performance and participation, the 'one' is the unified one and the 'many' are the individuals, as opposed to the contemporary Western notion of the 'one' being the individual and the 'many' being the group (Bolt 2004:139). In methexis, the 'one' symbolises a cosmic entity that can only be experienced through the many and, according to Carter (1996:71), is at the heart of all ritual and ceremony in Aboriginal culture (Bolt 2004:139; see also Durkheim 1915; Morphy 1991:116; Taylor 1996:103).

\section{Site 10041}

In Figure 4.12, three different types of long-necked spearthrowers are depicted. The two main human-like figures hold rectilinear sub-types. The body of each spearthrower has a cross-hatched design that matches the body covering of the male figures. In the horizontal plane, a curvilinear

\section{terra australis 47}


long-necked spearthrower lies next to a barbed spear; to the left of the panel, a triangular-shaped long-necked spearthrower stands vertically next to a very short three-pronged spear. This last spearthrower defies the existing taxonomy but was included in the multi-sided sub-category for want of a better place. Here there are three identifiable layers of painting:

- The lowest layer with a faded double-line human-like figure being superimposed by the figures enhanced in blue.

- An intermediate layer with two other figures (one partially painted) and an oval-shaped crosshatched curvilinear spearthrower and bokko spear, indicated in blue and superimposed over the faded figures but under the black layer.

- The top layer consisting of the human-like figures and artefacts enhanced in black.

All these images are painted in shades of red pigment. The image includes a rare depiction of a dog-like animal (dingo?) and a smaller figure, which I read as a probable child who appears to carry an unidentified bundle on its head. The large male figure just in front of the 'child' holds two short square-barbed spears in one hand and has a tassel or small bag hanging from his neck. The other large male figure near the front has two baubled tassels hanging from his neck. It is unclear if the cross-hatched shapes over the bodies of the two large male figures are capes, long bags, some other covering or simply a decorative convention for the torso.

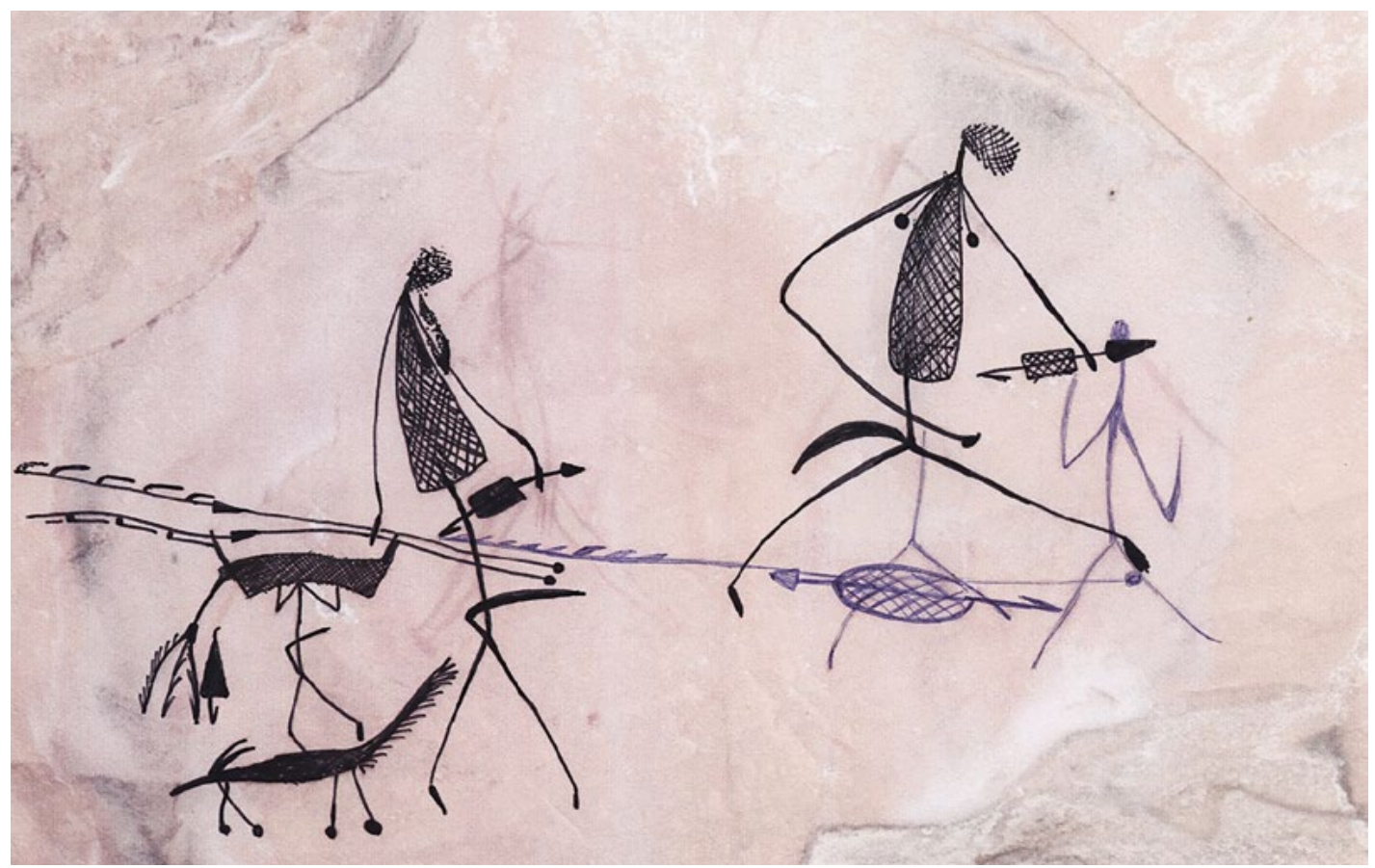

Figure 4.12 Human-like figures with a dog-like animal and items of material culture, from site 10041.

Source: Photograph by Duncan Wright, enhancement by John Hayward.

The manner in which these human-like figures are painted does not conform to any particular common or recognised style for the region, but has highly stylised features such as curved torsos, thin, tapering limbs that are thickest at the top, delicate hands and feet, fine-hatched infill and attention to the details of material culture items. The individuality expressed in this image conforms on a number of levels to previous images, but exudes a uniqueness of manner and deftness with the brush and pigments. This singularity and deftness appears to signal the personal style of the artist and one that is identifiable by the subtleness of the lines, the repetition of texture and the inventiveness of spearthrower diversity. 


\section{Spearthrower construction}

One major variation in the painting of spearthrowers in the rock art is that some are depicted with solid infill and others are depicted with distinctive patterns and outlines. Such differentiation could indicate structural variations in the construction of actual 'real world' artefacts, with some being made from solid materials and others from linear or 'open' materials. The other possibility is that the linear patterns suggest incised or painted decorations on a solid surface. Lewis (1988:62) speculated that the 'fine line-control and carefully executed patterns inside the round or squared sections of some examples suggest that its shape was accurately rendered', although he also recognised that some artistic licence may have influenced the depictions. The construction methods of such items are worth considering, especially if depictions of longnecked spearthrowers represent a transition from broad to long spearthrower types.

The possible solid construction method of some long-necked spearthrowers could have been similar to broad spearthrower types that were presumably shaped from single pieces of timber, in much the same way as ethnographic spearthrower examples from central Australia, which also have wide bodies, and long-notched lath spearthrowers from Arnhem Land and are commonly seen in museum collections. Previously, there was little conclusive ethnographic evidence of any broad spearthrower types from Arnhem Land, but during the June 2013 survey of the southern section of Djawumbu Madjawarrnja massif a stencil of a spearthrower that fits more comfortably into the category of a solidbodied broad type rather than a long type spearthrower was discovered (Figure 4.13). Adding to the evidence, I recently came across two broad spearthrowers at Museum Victoria, collected by Baldwin Spencer from the Roper River region of eastern Arnhem Land, which is approximately $300 \mathrm{~km}$ from the painting sites. These artefacts are morphologically similar to the aforementioned stencilled broad spearthrower from the Djawumbu Madjawarrnja massif, and were described in Spencer's notes as 'short, broad and spatulate' (Figure 4.13). The handle, body and hook extensions have each been shaped from a single piece of softwood; the hook itself is a harder timber piece that had been attached to the neck with resin. It is possible that the solid-infilled long-necked spearthrower types evident in the rock art were also made in a similar fashion to the broad spearthrowers from the Roper River collected by Spencer, but alternatively it is possible that they were made as two-piece constructions, with the basic central shaft used to attach a solid body made from a separate, shaped piece of timber or bark.
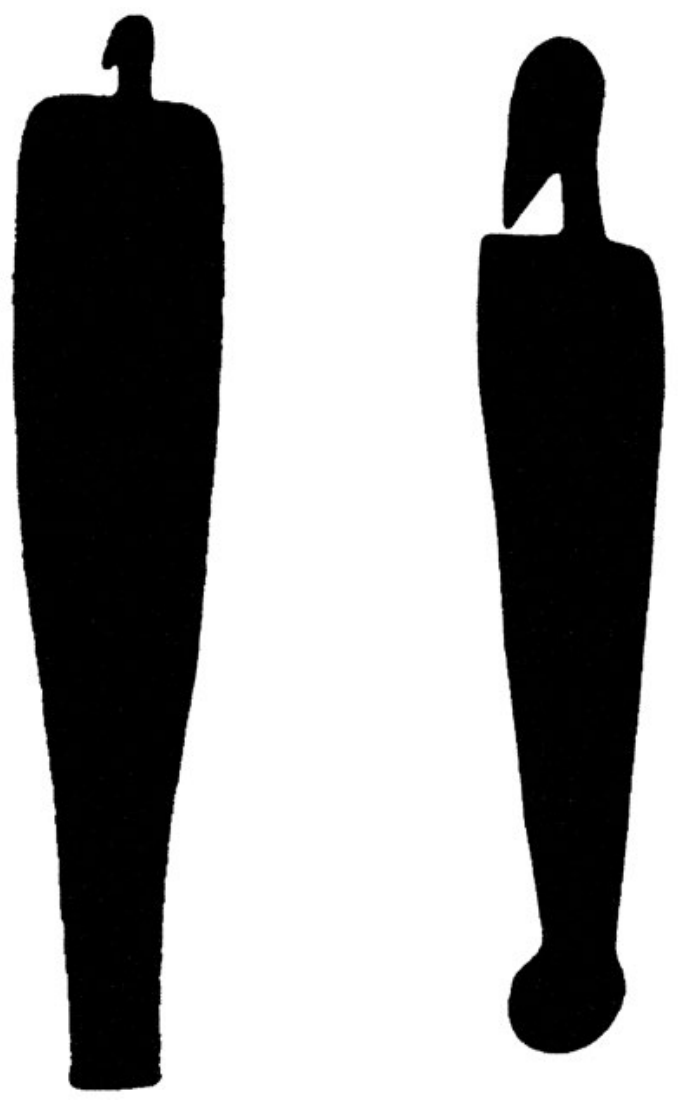

Figure 4.13 Two broad spearthrowers.

Left: drawing of a broad spearthrower collected by Baldwin Spencer from the Roper River region. Right: solid infill interpretation of a stencil from the Djawumbu Madjawarrnja massif. Not to scale.

Source: John Hayward. 
The construction methods of the more common 'open' construction long-necked spearthrower type is speculative, as there are no stencilled or ethnographic examples for comparison. There appears to be a basic hooked stick central shaft, which resembles a cylindrical spearthrower, onto which was added an elaborate body form that could have been made from shaped or natural timber, bamboo or reed and infilled with similar materials or other fibres held together with twine and resin. The construction could have been quite light but also quite fragile, and probably would have had a very short life span unless it was purely for display.

One painted example of a possibly 'open' construction long-necked spearthrower (Figure 4.14) depicts a single concave-sided spearthrower by itself. The handle of the artefact is a diamond shape and displays the central shaft of the spearthrower. The same shaft extends through the centre of the body and protrudes beyond the top end for a short distance before the hook is formed at the distal end. Tassels of some sort have been added to the hook and handle. The body appears to have been constructed from four pieces of shaped material joined at the corners in an unspecified way. The sides are made of curved forms, not dissimilar to boomerangs, the right-hand side having a more pronounced curve than the left. The shape of this example is not dissimilar to others in the Type $\mathrm{C}$ classification of longnecked spearthrowers, but is proportionally wider and the curves are more exaggerated. Whether this painting truly depicts an open structure, or a solid form with a defined pattern applied to the surface, is unclear, although the continuation of the central shaft through the body suggests the former.

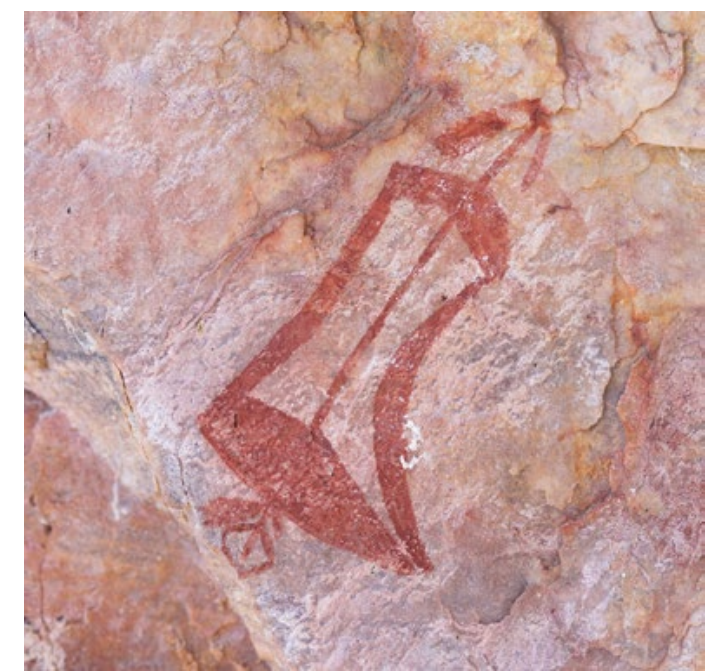

Figure 4.14 Painting of a concave-sided longnecked spearthrower, from site R10018.

Source: Photograph by lain Johnston.

It is not difficult to speculate on how a broad, solid spearthrower form could have evolved into a narrower, long shape. This transformation might have followed, or driven, the transition from heavier and slower single-piece hardwood spears to the lighter and faster bamboo and reed-shafted spears. These spears were made from the Bambusa arnhemica and Phragmites karka, species that proliferated during the influx of freshwater wetlands in the last 1500 years, and required a lighter but longer spearthrower technology for propulsion (Cundy 1989). This apparent change in the spear/spearthrower technology, from high-energy and low-velocity to lowenergy and high-velocity technology systems highlights the anomaly of the long-necked spearthrower, which plays no obvious part in that transition. The introduction of a large and complex-shaped central body, whether of solid or 'open' construction, was clearly not an essential, or perhaps even desirable, addition to basic spearthrower design or function. Rather, other reasons for the existence of longnecked spearthrowers in rock art are worthy of discussion. 


\section{Symbology of the long-necked spearthrower}

Long-necked spearthrowers in rock art are associated with a range of material culture items, all of which can be recognised from ethnographic collections and are, therefore, considered as artefacts that belong to one of the more recent rock art traditions. Long-necked spearthrowers, however, have no known ethnographic equivalents, and so the only 'proof' of their existence is as rock art depictions.

Besides a lack of ethnographic evidence, there are other strong arguments against the existence of these cultural items in the material world that are based on the technological question of their functional benefit for spear-throwing. There is seemingly nothing about the size, shape and form of the spearthrower design that would improve the efficiency of the technology and, conversely, the large body of the implement would actually hinder practical spear-launching. Carrying such a large item would be difficult and impractical, plus there would need to have been a substantial investment of time and energy to make each item, which, because of its fragility, may have had a very short lifespan. A conclusion of such logic is that these items have little or no sociotechnical function and, therefore, did not exist as everyday artefacts.

It can be argued, however, that such artefacts had some symbolic (e.g. totemic) reference or other such cultural significance, and that the uniqueness of each spearthrower emblem was an expression of personal or group identity emanating from its display at public events such as ceremonies, as well as during personal use (Taylor 1996:104). The elaboration of the spearthrower form would also have had a socio-ideological function that allowed for a demonstration of construction and carving skill by an individual maker (Dobres 1995:41). Such items may also have had unique values that prevented them being traded or exchanged to anthropologists and collectors during early encounters, thus ensuring their non-appearance in ethnographic collections.

Any of the above reasons could explain the existence of long-necked spearthrowers as items with ideological functionality that came into fashion or customary use at a particular time in 'recent' history, but they do not adequately explain why long-necked spearthrowers fell out of fashion and did not survive through to ethnographic times along with the large number of other items such as certain barbed spears, goose-wing fans and other spearthrower types with which they were concurrently depicted. One explanation for this is that they only existed as graphic signatures in rock art and were still used as such until colonial times at which time other concerns and influences prevailed. If this is the case, then long-necked spearthrowers as items of material culture are a depicted fiction, or at least characters in a fiction who act in a number of roles to help render a story. Their agency as fictional characters is not unreasonable as long-necked spearthrowers project a uniqueness and individualism at least equal to the human-like depictions they associate with. Long-necked spearthrower agency, as I argue here, is not so much sociotechnical as socio-ideological and, as such, has no reducible bearing upon the technical world. The performative actions of long-necked spearthrowers, then, are largely limited to the socioideological and, as far as we know, to the symbolic realm of rock art where they were created and where they share their individuality with the human-like figures they associate with, creating the illusion that it is the human-like being who has the individuality.

This relationship between human beings and artefacts is already complex in the real material world as people and material culture share linked histories, shared intentions, distributed agency and connected subliminal influences (David 2002:211-213; Dobres 2000; Gosden 1999:120; Introna 2012:35; Latour 2003:39). In the realm of rock art, relationships between human actors and material culture become even more complex, as the rock art motif is also an artefact and in this sense a depiction of a long-necked spearthrower is an artefact within an artefact. While it may be apparent that the image of a long-necked spearthrower has agency as painting - i.e. as a 
representation of individuality - it seems that its agency is not confined to the painted realm, for we, the viewers, are also drawn into its influence. In the real world an artefact, as technology, behaves as 'a way of revealing' (Heidegger 1977:12). In this hypothesis, the artefact comes into being not because the artisan manufactured it but because the concept of the artefact reveals itself to the artisan through the realm of revealing and unconcealment (Heidegger 1977:13; see also David 2002:211-212). It is this shared responsibility, or what Barad (2003:814) calls 'intra-action' and Introna (2012:39) calls 'co-constitutive agency', which infuses the social with the technical, and the maker with the artefact, and which brings the artefact into being. If the artefact exists only in the realm of rock art, the same applies, except that here the revealing of the artefact (the rock art motif) comes about through the social and the ideological.

Consequently, it appears that in western Arnhem Land the long-necked spearthrower is an artistic invention whose function is that of a signifier of both individual and group identity in and via rock art motifs. In many cases, the morphology of long-necked spearthrowers in rock art reference all other main spearthrower types found in both rock art and ethnographic collections, but this is not evidence that they existed in both worlds. The assumption that because an image appears in rock art means it must have also existed in reality results from the inter-tangled realm of rock art with the so-called real material world, and is no more true than the idea that every item that existed in the real material world was painted in rock art. These artefacts were probably painted during the recent chronological rock art period of the region at a time when cylindrical, broad and long-notched lath spearthrowers all existed together as functioning spearthrower technologies. The artists that painted these images during that period appear to have used the long-necked spearthrower as a device to convey both the individuality of their paintings and of themselves.

\section{Conclusion}

The diversity, creative inventiveness and ideological scope of the long-necked spearthrower seems unmatched by other depictions of material culture items in western Arnhem Land rock art, and appears to have no equivalent in ethnographic material culture collections from the wider region. More than half the long-necked spearthrowers recorded for this study are unique representations of the spearthrower type; the rest exhibiting variations on the original themes. Paintings with multiple long-necked spearthrowers, each with individual designs, appear to reinforce the individuality of each human-like figure they are associated with. Those images, on the other hand, that have many, choreographed human figures all holding similarly shaped long-necked spearthrowers, suggest group solidarity. I argue, therefore, that a principal function of long-necked spearthrowers is that of an agent within the graphic symbology of the rock art of a discrete region of western Arnhem Land.

Those long-necked spearthrower depictions, which appear as solid forms, appear to reference the morphology of broad spearthrowers; others have similarities to cylindrical and long-notched lath spearthrowers. In such cases, rock art depictions of long-necked spearthrowers could be considered as having material connections with documented ethnographic artefact types, and in this sense have one foot in the material world and one in the symbolic. The existence of broad, cylindrical and long-notched lath spearthrower types in ethnographic collections, and the morphological connection of long-necked spearthrowers with all types through rock art alone, complicates Lewis's chronology that suggested a material world transition from broad to long types some 1000-2000 years ago, with the long-necked spearthrower as an intermediary experimental form or aberration. I suggest that all these spearthrower types existed side-by-side, for a period before European contact, as items with separate functions in the material world: 
the broad and cylindrical spearthrowers, associated with heavier composite and one-piece spears; the long-notched lath spearthrower, used exclusively for a range of lighter hafted spears; and long-necked spearthrowers, as mediating agents between the material and graphic worlds, not reducible to technological functions but rather having a wide-ranging ideological function.

\section{Acknowledgements}

The data for this chapter were collected during the 2012-2014 fieldwork surveys conducted for the Mirarr Gunwarddebim (rock art) project. The project was initiated and is supported by the Mirarr community and the Gundjeihmi Aboriginal Corporation (GAC). I would like to thank all GAC board members, in particular Yvonne Margarula and GAC Chief Executive Officer Justin O'Brien for their ongoing support. Thanks also to Kakadu National Park, Natural Cultural Programs Unit and Energy Resources of Australia mining for access to survey areas including Jabiluka mineral lease. Special thanks are also extended to all members of the Mirarr Gunwarddebim survey teams (too many to mention), but in particular Sally May as co-ordinator, Paul Taçon as mentor, Phil Davill (Mirarr Gunwarddebim team member) for maps and technical support and Iain Johnston (The Australian National University) for companionship. I am grateful to Darrell Lewis (University of New England) for giving me an electronic copy of his 1988 monograph that has been used extensively for this chapter. This chapter benefited from input and comments from my friend Ian Hamilton, Sally May (Griffith University), Susanne Schech (Flinders University), the editors of this publication Paul Taçon and Bruno David and independent reviewers.

\section{References}

Allen, H. and K. Akerman 2015. Innovation and change in northern Australian Aboriginal spear technologies: The case for reed spears. Archaeology in Oceania. 50:82-92. doi.org/10.1002/arco.5051

Barad, K. 2003. Posthuman performativity: Towards an understanding of how matter comes to matter. Signs 28(3):801-831. doi.org/10.1086/345321

Bolt, B. 2004. Art beyond Representation: The Performative Power of the Image. I.B. Tauris, London.

Brandl, E.J. 1973. Australian Aboriginal Paintings in Western and Central Arnhem Land: Temporal Sequences and Elements of Style in Cadell River and Deaf Adder Creek Art. Australian Institute of Aboriginal Studies, Canberra. (2nd edition 1988.)

Carter, P. 1996. The Lie of the Land. Faber and Faber, London.

Chaloupka, G. 1984. From Palaeoart to Casual Paintings: The Chronological Sequence of Arnhem Land Plateau Rock Art. Monograph Series 1. Northern Territory Museum of Arts and Sciences, Darwin.

Chaloupka, G. 1993. Journey in Time: The World's Longest Continuing Art Tradition. Reed, Chatswood.

Cundy, B.J. 1989. Formal Variation in Australian Spear and Spearthrower Technology. British Archaeological Reports International Series 546, Oxford.

David, B. 2002. Landscapes, Rock Art and the Dreaming: An Archaeology of Preunderstanding. Leicester University Press, Leicester.

Davidson, D.S. 1934. Australian spear-traits and their derivations. Journal of the Polynesian Society $43: 41-72$ 
Davidson D.S. 1936. The spearthrower in Australia. Proceedings of the American Philosophical Society LXXVI:445-483.

Dobres, M. 1995. Gender and prehistoric technology: On the social agency of technical strategies. World Archaeology 27(1):25-49. doi.org/10.1080/00438243.1995.9980291

Dobres, M. 2000. Technology and Social Agency. Blackwell, Oxford.

Durkheim, E. 1915. The Elementary Forms of the Religious Life. Allen and Unwin, London.

Gosden, C. 1999. Anthropology and Archaeology: A Changing Relationship. Routledge, Florence.

Hamby, L. 2010. Containers of Power. Utber and Patulio, Richmond.

Haskovec, I.P. 1992. Northern Running Figures of Kakadu National Park: A study of regional style. In J. McDonald and I.P. Haskovec (eds), State of the Art: Regional Rock Art Studies in Australia and Melanesia, pp. 140-158. Occasional AURA Publication 6. Australian Rock Art Research Association, Melbourne.

Heidegger, M. 1977. The Question Concerning Technology and Other Essays. Garland, New York.

Introna, L.D. 2012. Towards a post-human intra-actional account of socio-technical agency (and morality). In P. Kroes and P. Verbeek (eds), The Moral Status of Technical Artefacts, pp. 31-53. Springer Netherlands, Dordrecht.

Latour, B. 2003. The promise of constructivism. In D. Ihde and E. Selinger (eds), Chasing Technoscience: Matrix for Materiality, pp. 27-46. Indiana University Press, Bloomington.

Lewis, D. 1988. The Rock Paintings of Arnhem Land, Australia: Social, Ecological and Material Culture Change in the Post-Glacial Period. British Archaeological Reports International Series 415, Oxford.

Lewis, D. 1996. In defence of Arnhem Land rock art research. Australian Archaeology 43:12-20. doi.org/10.1080/03122417.1996.11681578

Morphy, H. 1991. Ancestral Connections: Art and an Aboriginal System of Knowledge. University of Chicago Press, Chicago.

Taçon, P.S.C. 1989. From Rainbow Snakes to 'X-Ray' Fish: The Nature of the Recent Rock Painting Tradition of Western Arnhem Land, Australia. Unpublished PhD thesis. The Australian National University, Canberra.

Taylor, L. 1996. Seeing the Inside: Bark Painting in Western Arnhem Land. Clarendon Press, Oxford.

Welch, D.M. 1997. Fight or dance? Ceremony and the spearthrower in northern Australian rock art. Rock Art Research 14(2):88-112.

Zeki, S. 2006. The neurology of ambiguity. In M. Turner (ed.), The Artful Mind: Cognitive Science and the Riddle of Human Creativity, pp. 243-270. Oxford University Press, Oxford. doi.org/10.1093/acpr of:oso/9780195306361.003.0013 
This text is taken from The Archaeology of Rock Art in Western Arnhem Land, Australia, edited by Bruno David, Paul Taçon, Jean-Jacques Delannoy and Jean-Michel Geneste, published 2017 by ANU Press, The Australian National University, Canberra, Australia. 